\section{Utilidad de los ensayos de liberación de interferón gamma para la detección de tuberculosis en población pediátrica}

\section{RESUMEN}

El ensayo de liberación de interferón gamma es un método novedoso para el diagnóstico de la tuberculosis aunque su utilidad es incierta en población pediátrica.

Objetivo: examinar la utilidad diagnóstica del QuantiFERON-TB Gold $\left(\mathrm{QFT}-\mathrm{G}^{\circledR}\right)$, uno de los ensayos de liberación de interferón gamma comerciales, en población pediátrica del Estado de Sonora, México.

Materiales y métodos: estudio transversal con 160 menores de 19 años de edad, en un hospital de Sonora, México. Se compararon tres grupos, uno de pacientes con tuberculosis activa, otro con tuberculosis latente y uno más de controles hospitalizados. Los criterios de Stegen-Toledo fueron el estándar empleado. Se usó regresión logística multivariada para evaluar la asociación entre el QFT-G ${ }^{\circledR}$ y los predictores seleccionados.

Resultados: la sensibilidad del QFT-G ${ }^{\circledR}$ en la tuberculosis activa fue de $76.9 \%$ y la especificidad de $90.3 \%$. Para la tuberculosis latente la sensibilidad fue $25.0 \%$ y la especificidad de $98.6 \%$. El QFT-G ${ }^{\circledR}$ mostró una concordancia moderada $(\mathrm{K}=0.574)$ con el estándar. También mostró asociación con la radiografía de tórax sugestiva de tuberculosis (razón de momios ajustada 4.37, IC 95\%: 1.42, 13.42) y con el hecho de ser caso de tuberculosis pulmonar (razón de momios ajustada 6.15, IC 95\%: 1.90, 19.92).

Conclusión: el ensayo de liberación de interferón gamma es un método útil para diagnosticar tuberculosis activa y puede emplearse para descartar tuberculosis latente en población pediátrica.

Palabras clave: ensayos de liberación de interferón gamma, tuberculosis en la infancia, diagnóstico.

\section{Interferon gamma release assays for diagnosis of tuberculosis in pediatric population}

\section{ABSTRACT}

The interferon-gamma release assay (IGRA) is a novel method for the diagnosis of tuberculosis (TB), but its usefulness is uncertain in children.

Objective: Examine the diagnostic capacity of QuantiFERON-TB Gold $\left(\mathrm{QFT}^{\mathrm{B}}{ }^{\circledR}\right)$ one of the IGRA commercial kits, in pediatric population of Sonora, Mexico.
Gerardo Álvarez-Hernández ${ }^{1}$ Jehan Bonizú Álvarez-Meza² María del Carmen Candía-Plata ${ }^{1}$ Enrique Bolado-Martínez ${ }^{3}$ Miriam Denisse García-Villa ${ }^{1}$ Manuel Alberto Cano-Rangel ${ }^{2}$ María de los Ángeles Durazo-Arvizu² Luis Fernando López-Soto ${ }^{1}$

${ }^{1}$ Departamento de Medicina y Ciencias de la Salud, Universidad de Sonora.

${ }^{2}$ Hospital Infantil del Estado de Sonora.

${ }^{3}$ Departamento de Ciencias Químico Biológicas, Universidad de Sonora.

Recibido: 22 de julio del 2015

Aceptado: 28 de octubre del 2015

Correspondencia: Gerardo Álvarez Hernández Departamento de Medicina y Ciencias de la Salud Universidad de Sonora galvarezh63@gmail.com

Este artículo debe citarse como Álvarez-Hernández G, Álvarez-Meza JB, CandíaPlata MC, Bolado-Martínez E, García-Villa MD, Cano-Rangel MA et al. Utilidad de los ensayos de liberación de interferón gamma para la detección de tuberculosis en población pediátrica. Acta Pediatr Mex 2015;36:442-455. 
Methods: Cross-sectional design with 160 subjects less than 19 years old, in a hospital in Sonora, Mexico. Three groups were compared: one from patients with active TB (TBA), another one with latent TB (TBL) and one of hospital controls. The Stegen-Toledo criteria were the gold standard used. Multivariate logistic regression was performed to test the association between QFT-G $^{\circledR}$ and selected predictors.

Results: The sensitivity of QFT-G ${ }^{\circledR}$ in TBA was $76.9 \%$ and specificity $90.3 \%$. For TBL, sensitivity was $25.0 \%$ and specificity of $98.6 \%$. QFT-G ${ }^{\circledR}$ showed a moderate agreement $(K=0.574)$ with the standard. QFT-G ${ }^{\circledR}$ was associated with a chest radiograph suggestive of TB, (OR adjusted $4.37,95 \% \mathrm{Cl}: 1.42,13.42)$ and being a case of pulmonary TB (OR adjusted $6.15,95 \% \mathrm{Cl}: 1.90,19.92)$.

Conclusion: The interferon-gamma release assay shows significant capacity to diagnose TBA and can be used to rule out TBL in the pediatric population.

Keywords: Interferon-gamma release assay, Tuberculosis in childhood, Diagnosis.

\section{INTRODUCCIÓN}

La tuberculosis es un problema de salud pública en gran parte del mundo. La Organización Mundial de la Salud estima que anualmente ocurren nueve millones de casos y fallecen 1.5 millones de personas debido a esta enfermedad. ${ }^{1}$ En México, en el 2014, se registraron 20137 casos de todas las formas de tuberculosis y fallecieron 2300 personas. En el país, Sonora ocupa el cuarto lugar en incidencia (32.0/100 000) con todas las formas de tuberculosis; misma posición que tiene en tuberculosis pulmonar con una tasa de 28.6/100 000, en ambos casos duplicando el promedio nacional. En mortalidad, el estado ocupa la segunda posición en todas las formas de tuberculosis $(4.7 / 100000)$ y en tuberculosis pulmonar (3.9/100 000), más del doble de la media nacional. ${ }^{2}$

Respecto a la tuberculosis pediátrica no hay precisión acerca de su magnitud real en el mundo; no obstante que su existencia refleja transmisión activa en población adulta ${ }^{3}$ se considera que ocurre en personas menores de 15 años de edad. En México, esta categoría incluye a personas menores de 19 años de edad.

De acuerdo con la Organización Mundial de la Salud ${ }^{1}$ en el 2013 ocurrieron, en todo el mundo, unos 550000 casos nuevos y unas 80000 defunciones; pero otras estimaciones sugieren que hasta $11 \%$ del total de casos corresponde a niños y en regiones pobres esta proporción se incrementaría hasta $15 \%,{ }^{4}$ mientras en países de alta endemia constituiría entre 30 y $40 \%$ de la carga total de tuberculosis. ${ }^{5}$ En México el Programa Nacional de Prevención y Control de la tuberculosis considera a personas menores de 19 años como casos pediátricos y estimó, en el 2014, que $8.2 \%$ (1 661) de todos los casos ocurrieron en este grupo. ${ }^{2}$

Las discrepancias en las cifras de tuberculosis pediátrica pueden obedecer a que su diagnóstico es complejo debido a la poca especificidad 
de sus síntomas, a la naturaleza paucibacilar en este grupo y a la dificultad que los niños tienen para expectorar, lo que puede reducir la capacidad diagnóstica de los métodos tradicionales como la baciloscopia o el cultivo. Por ejemplo, se ha estimado que únicamente entre 20 y $40 \%$ de los cultivos de esputo desarrollan Mycobacterium tuberculosis. ${ }^{3} \mathrm{Nicol}$ y sus colaboradores ${ }^{6}$ señalaron que la sensibilidad de la baciloscopia es menor a $15 \%$, por lo que se ha recomendado evaluar técnicas de respuesta rápida para identificar al bacilo. ${ }^{1,7,8}$ Una de esas pruebas es el ensayo de liberación de interferón gamma (IGRA por sus siglas en inglés) que mide la producción de interferón gamma en respuesta a la estimulación de antígenos específicos de M. tuberculosis (ESAT-6, CFP10 y TB 7.7). ${ }^{9,10}$ Uno de los ensayos comerciales de mayor uso en el mundo es el QuantiFERONTB Gold $\left(\mathrm{QFT}_{-} \mathrm{G}^{\circledR}\right)$, que ha mostrado ser útil en población adulta pero persiste la incertidumbre acerca de su valor para detectar la infección en niños, particularmente en regiones con elevadas tasas de transmisión. ${ }^{9-11}$ En México, el método ha mostrado ser válido en poblaciones confinadas, ${ }^{12}$ además de ser costo-eficaz para el diagnóstico de la tuberculosis en adultos. ${ }^{13}$ Poco se conoce acerca de su valor en niños mexicanos aunque ha mostrado su utilidad para el diagnóstico de tuberculosis latente ${ }^{14}$ $y$, recientemente, en migrantes que residen en Estados Unidos. ${ }^{15}$

\section{OBJETIVO}

Examinar la utilidad diagnóstica del QFT-G $^{\circledR}$ en pacientes menores de 19 años de edad, con sospecha de tuberculosis, que recibieron atención médica en un hospital pediátrico del Estado de Sonora, México. La capacidad diagnóstica de la prueba se comparó con los criterios de Stegen-Toledo, un conjunto de datos clínicos y epidemiológicos de amplio uso en pediatría ${ }^{16}$ que fueron usados como estándar de referencia ante las dificultades para confirmar bacteriológicamente la enfermedad en este grupo poblacional.

\section{MATERIALES Y MÉTODOS}

Mediante un estudio transversal se evaluaron la validez y la confiabilidad del reactivo QFT-G ${ }^{\circledR}$, en muestras de sangre venosa periférica, para identificar infección tuberculosa en pacientes de un hospital público pediátrico del Estado de Sonora (entre el 1 de enero del 2012 y el 30 de junio del 2013). El estudio recibió financiamiento del Consejo Nacional de Ciencia y Tecnología. Antes de su implementación recibió la aprobación de dos comités de ética en investigación.

El criterio para elegir a los pacientes para el estudio tomaba en cuenta si tenían hasta 18 años de edad; se contó con el consentimiento informado y signado por los familiares responsables; tenían residencia por lo menos de 6 meses antes de la entrevista en alguna localidad de Sonora; no padecían o habían padecido alguna infección viral, por ejemplo, varicela, sarampión por referencia materna o del tutor. Fueron excluidos los que tenían alguna inmunodeficiencia o enfermedades renales, hepatopatías y padecimientos hematooncológicos, y si recibían o habían recibido tratamiento inmunodepresor o antituberculoso durante los dos meses previos a la fecha de determinación mediante QFT-G ${ }^{\circledR}$.

Se estudió una muestra de probabilidad en 160 sujetos (poder estadístico $=0.86$ ) elegidos convenientemente de las áreas de hospitalización. Médicos residentes de pediatría y nutriólogas, adiestrados en los procedimientos del estudio pero ignorantes respecto a su propósito, obtuvieron información clínica y epidemiológica mediante un cuestionario estructurado, cuyos datos fueron empleados para clasificar a los sujetos con probable tuberculosis latente o activa, así como en un grupo control. La clasificación fue como se describe a continuación. 
Grupo I: pacientes con probable tuberculosis activa

Pacientes con cuadro respiratorio de 2 o más semanas de evolución acompañado por una o más de las siguientes características: a) no mejorar con los tratamientos antibióticos dirigidos a los patógenos habituales; b) tener radiología con imágenes sugestivas de tuberculosis; c) antecedente de contacto 4 horas diarias o más con un paciente sospechoso o confirmado de tuberculosis; d) reacción cutánea positiva ( $\geq 5 \mathrm{~mm}$ de induración cutánea 72 horas después de la aplicación) a la intradermorreacción con derivado proteínico purificado.

Grupo II: pacientes con probable tuberculosis latente

Pacientes sin evidencia clínica, bacteriológica ni radiológica de tuberculosis que tuvieron una reacción cutánea positiva al derivado proteínico purificado $\geq 5 \mathrm{~mm}$ de induración cutánea a las 72 horas de su aplicación) que pudieron tener contacto con un tuberculoso.

\section{Grupo III: pacientes sin patología respiratoria} aparente

Pacientes hospitalizados, sin datos de patología respiratoria aguda o crónica y sin contacto sustancial (4 horas o más diarias) reciente con un paciente sospechoso o confirmado de tuberculosis.

\section{Recolección de muestras sanguíneas}

A los sujetos elegidos se les tomó una muestra de $3 \mathrm{~mL}$ de sangre venosa periférica que se depositó en tubos específicos para el ensayo de liberación de interferón gamma $\left(\mathrm{QFT}-\mathrm{G}^{\circledR}\right)$. Los tubos fueron transportados de inmediato a $22^{\circ} \mathrm{C}( \pm 5$ grados) a un laboratorio especializado y fueron procesados dentro de las 16 horas posteriores a la toma de la muestra. El ensayo $\mathrm{QFT} \mathrm{G}^{\circledR}$ fue procesado de acuerdo con las guías de uso. ${ }^{17}$ Tras una incubación de 16 a 24 horas, a $37^{\circ} \mathrm{C}$, se midió el interferón- $\gamma$ liberado en el plasma por los linfocitos activados mediante un ensayo de tipo ELISA. Los datos de absorción fueron convertidos a unidades internacionales por mililitro $(\mathrm{UI} / \mathrm{mL})$ utilizando una curva estándar para cada placa de ELISA, misma que fue procesada por duplicado y aceptada sólo si el coeficiente de correlación fue mayor de 0.98 y el coeficiente de variación menor de 15\%. Este método tiene un límite de detección de $0.05 \mathrm{UI} / \mathrm{mL}$. Los datos individuales sólo se aceptaron como válidos si una respuesta (al menos a un antígeno: TB7.7, ESAT-6, CFP-10) fue mayor o igual a $0.35 \mathrm{UI}$ $\mathrm{mL}$ bv y la concentración de IFN- $\gamma$ fue 2.5 veces mayor que la del blanco.

\section{Aplicación del derivado proteínico purificado}

La aplicación se efectuó cuando menos 72 horas antes de la toma de muestra para el QFT$\mathrm{G}^{\circledR}$ mediante la técnica de Mantoux. Se utilizó derivado proteínico purificado RT23 (Statens Serum Institut-Batch 1530-A) en ampolletas de $1 \mathrm{~mL}$ equivalente a 10 dosis de $0.1 \mathrm{~mL}$ con dos unidades de tuberculina de derivado proteínico purificado. La medición final de la dermorreacción se realizó 72 horas después a la aplicación y fue efectuada por un médico distinto al que la aplicó, para reducir el sesgo de observación. La interpretación de la prueba se basó en el diámetro de la induración de acuerdo con los siguientes criterios: a) si fue menor de $5 \mathrm{~mm}$ se considero como negativa; b) si fue de $5 \mathrm{~mm}$ o más se interpretó como una reacción positiva. ${ }^{18}$

\section{El estándar de referencia: los criterios de Stegen-Toledo}

La situación clínica de los sujetos con sospecha de tuberculosis se estableció mediante el empleo de los criterios de Stegen-Toledo. ${ }^{16}$ El sistema 
usa una medición que establece una certeza diagnóstica cuando se tienen 7 o más puntos; si se obtienen entre 5 y 6 puntos se acepta que el diagnóstico es factible y amerita iniciarse el tratamiento; entre 3 y 4 puntos se considera que el diagnóstico es posible y amerita estudio profundo; con menos de 2 puntos la sospecha de tuberculosis se descarta. Este sistema fue el estándar de referencia empleado para evaluar el desempeño del QFT-G ${ }^{\circledR}$. Los criterios empleados para la clasificación de los sujetos incluyeron: a) hallazgo de bacilos ácido-alcohol resistentes ( 7 puntos); b) granuloma específico por histología (4 puntos); c) derivado proteínico purificado mayor de $10 \mathrm{~mm}$ (3 puntos); d) antecedente epidemiológico (2 puntos); e) radiografía sugestiva (2 puntos); f) cuadro clínico sugestivo (2 puntos).

\section{Análisis estadístico}

Se efectuó estadística descriptiva y las diferencias entre los grupos se examinaron con prueba polinominal de $\chi^{2}$ (variables categóricas) y ANOVA (variables continuas). Se probaron hipótesis a dos colas y fueron considerados estadísticamente significativos valores de $p<0.05$. Para estimar la validez clínica del QFT-G ${ }^{\circledR}$ se compararon los resultados de la prueba de los pacientes con el estándar seleccionado. El resultado del QFT-G ${ }^{\circledR}$ se consideró positivo cuando fue $\geq 0.35 \mathrm{UI} / \mathrm{mL}$. La validez del método se estimó con el cálculo de la sensibilidad y la especificidad para los grupos con tuberculosis activa y latente, en tanto la confiabilidad fue examinada con el coeficiente de Kappa (K); para su interpretación se usaron los valores propuestos por Landis. ${ }^{19}$

Para examinar la relación entre una prueba positiva de QFT-G ${ }^{\circledR}$ y los predictores clínicos y sociodemográficos identificados en la etapa descriptiva se llevó a cabo una regresión logística multivariada. Se utilizó el procedimiento de eliminación en reversa para elegir las variables que ingresaron al modelo final. Se obtuvieron razones de momios ajustadas y sus respectivos intervalos de confianza a 95\%. La razón de probabilidades de $\chi^{2}$ fue empleada para examinar la significación del modelo final. Todo el análisis estadístico se realizó con el paquete NCSS $2007^{\circledR}$.

\section{RESULTADOS}

\section{Reproducibilidad de la prueba diagnóstica}

La reproduccibilidad del ensayo de liberación de interferón gamma fue confirmada al observar que se alcanzó una curva estándar de 8 puntos, por triplicado, con diluciones seriadas del estándar contenido en el equipo QFT-G ${ }^{\circledR}$ entre concentraciones de 0 a $8 \mathrm{UI} / \mathrm{mL}$. El coeficiente de determinación $R^{2}>0.9979$ demostró la precisión intraensayos. El coeficiente de variación que se obtuvo entre las repeticiones fue de $2.6 \%$, mucho menor al aceptable para el inmunoensayo enzimático (ELISA) donde uno $<7 \%$ es aceptable; con ello se demostró la precisión interensayos. La exactitud del método se demostró mediante ensayos múltiples con el control positivo en los que se obtuvo un coeficiente de variación $<0.05 \%$ con relación a la concentración de referencia.

\section{Descripción de los sujetos de estudio}

La muestra se constituyó con 160 sujetos, 52 de ellos clasificados en el grupo de tuberculosis activa, 36 en el de tuberculosis latente y 72 controles. La descripción de los factores sociodemográficos explorados se despliega en el Cuadro 1. La edad promedio de los sujetos fue de $6.8 \pm 0.71$ años sin diferencias significativas $(p=0.275)$ entre los grupos. En la determinación cuantitativa del QFT-G ${ }^{\circledR}$ se apreció que los valores promedio (en $\mathrm{UI} / \mathrm{mL}$ ) del grupo de tuberculosis activa $(5.00 \pm 0.72)$ fueron superiores a los de sujetos con tuberculosis latente y a los de los controles $(p<0.001)$. Con respecto a la induración pro- 
Álvarez-Hernández G, et al. Liberación de interferón gamma para detectar tuberculosis pediátrica

Cuadro 1. Características sociodemográficas seleccionadas de niños con sospecha de infección tuberculosa de un hospital pediátrico del estado de Sonora (2012-2013)

\begin{tabular}{|c|c|c|c|c|}
\hline Variable & TBA $(n=52)$ & $\begin{array}{c}\text { Número }(\%) \\
\text { TBL }(n=36)\end{array}$ & Controles $(n=72)$ & $p^{1}$ \\
\hline \multicolumn{5}{|l|}{ Sexo } \\
\hline Femenino & $29(55.8)$ & $14(38.9)$ & $27(37.5)$ & $<0.01$ \\
\hline Masculino & $23(44.2)$ & $22(61.1)$ & $45(62.5)$ & $<0.01$ \\
\hline \multicolumn{5}{|l|}{ Edad (en años) } \\
\hline 0 a 4 & $25(48.1)$ & $17(47.2)$ & $27(37.5)$ & $<0.01$ \\
\hline 5 a 9 & $6(11.5)$ & $14(38.9)$ & $20(27.8)$ & $<0.01$ \\
\hline 10 a 14 & $17(32.7)$ & $3(8.3)$ & $20(27.8)$ & 0.001 \\
\hline 15 a 19 & $4(7.7)$ & $2(5.6)$ & $5(6.9)$ & 0.108 \\
\hline \multicolumn{5}{|l|}{ Municipio de residencia } \\
\hline De alta endemia $(\geq 18.2 / 100,000)$ & $51(98.08)$ & $35(97.22)$ & $64(88.89)$ & 0.015 \\
\hline De baja endemia $(<18.2 / 100,000)$ & $1(1.92)$ & $1(2.78)$ & $8(11.11)$ & 0.007 \\
\hline \multicolumn{5}{|l|}{ Hacinamiento $^{2}$} \\
\hline Sí & $42(80.8)$ & $20(55.6)$ & $47(65.3)$ & $<0.01$ \\
\hline No & $9(17.3)$ & $14(38.9)$ & $24(33.3)$ & $<0.01$ \\
\hline Se desconoce & $1(1.9)$ & $2(5.5)$ & $1(1.4)$ & 0.325 \\
\hline \multicolumn{5}{|l|}{ Lengua indígena } \\
\hline Sí & $6(11.5)$ & $1(2.8)$ & $4(5.6)$ & 0.321 \\
\hline No & $46(88.5)$ & $33(91.7)$ & $68(94.4)$ & $<0.01$ \\
\hline Se desconoce & 0 & $2(5.5)$ & 0 & ns \\
\hline \multicolumn{5}{|l|}{ Escolaridad madre } \\
\hline Algún grado de primaria y menos & $13(25.0)$ & $4(11.1)$ & $15(20.8)$ & 0.040 \\
\hline Algún grado de secundaria y más & $39(75.0)$ & $30(83.3)$ & $54(75.0)$ & 0.028 \\
\hline Se desconoce & $00(00.0)$ & $2(5.6)$ & $3(4.2)$ & 0.655 \\
\hline \multicolumn{5}{|l|}{ Ocupación materna } \\
\hline Jornalera, obrera ${ }^{A}$ & $15(28.9)$ & $4(11.1)$ & $13(18.1)$ & 0.040 \\
\hline Hogar & $32(61.5)$ & $26(72.2)$ & $42(58.3)$ & 0.141 \\
\hline Otro $^{B}$ & $2(3.8)$ & $4(11.1)$ & $12(16.7)$ & 0.009 \\
\hline Se desconoce & $3(5.8)$ & $2(5.6)$ & $5(6.9)$ & 0.497 \\
\hline \multicolumn{5}{|l|}{ Escolaridad padre } \\
\hline Algún grado de primaria y menos & $22(42.3)$ & $5(13.9)$ & $21(29.2)$ & 0.003 \\
\hline Algún grado de secundaria y más & $20(38.5)$ & $26(72.2)$ & $40(55.5)$ & 0.025 \\
\hline Se desconoce & $10(19.2)$ & $5(13.9)$ & $11(15.3)$ & 0.304 \\
\hline \multicolumn{5}{|l|}{ Ocupación paterna } \\
\hline Jornalero, obrero $^{A}$ & $28(53.9)$ & $19(52.8)$ & $33(45.8)$ & 0.151 \\
\hline Desempleado & $3(5.8)$ & $3(8.3)$ & $3(4.2)$ & 1.000 \\
\hline Otro $^{B}$ & $11(21.1)$ & $9(25.0)$ & $21(29.2)$ & 1.000 \\
\hline Se desconoce & $10(19.2)$ & $5(13.9)$ & $15(20.8)$ & 0.082 \\
\hline \multicolumn{5}{|l|}{ Vive en pobreza ${ }^{3}$} \\
\hline Pobre & $10(19.2)$ & $2(5.5)$ & $19(26.4)$ & 0.001 \\
\hline Otro & $42(80.8)$ & $33(91.7)$ & $53(73.6)$ & 0.095 \\
\hline Se desconoce & $0(00.0)$ & $1(2.8)$ & $0(00.0)$ & ns \\
\hline
\end{tabular}

TBA: tuberculosis activa; TBL: tuberculosis latente.

${ }^{1}$ Basado en una prueba multinomial de $\chi^{2}$ para igualdad de proporciones.

²Duermen 2.5 o más personas por habitación destinada a dormir (Instituto Nacional de Geografía y Estadística, 2010).

${ }^{3}$ Se consideró que vivía en pobreza si el ingreso familiar mensual estaba por debajo de la línea de bienestar del CONEVAL y alguno de sus padres, o ambos, tenía algún grado de primaria o sin educación. La línea de bienestar fue de \$1,921.74 M.N. por mes [urbano] o menos de \$1,202.80 M.N. [rural]. Línea de bienestar de la Comisión Nacional de Evaluación de la Política de Desarrollo Social (CONEVAL). Ancluye adicionalmente otras ocupaciones como vendedor ambulante, empleada doméstica, peones, trabajadores de reparaciones. BIncluye empleados, técnicos, oficinistas y profesionistas. 
ducida por el derivado proteínico purificado, el grupo de tuberculosis latente mostró la mayor reactividad dérmica (14.86 $\pm 1.65 \mathrm{~mm}$ ), seguido por los del grupo con tuberculosis activa y por los del grupo control $(p<0.001)$. En el Cuadro 2 se muestran los detalles de esta distribución.

Se apreció que la tuberculosis pulmonar fue la forma clínica más frecuente de tuberculosis. De los 52 pacientes con la forma activa 41 (78.9\%) tuvieron tuberculosis pulmonar; 11 (21.1\%) tuvieron alguna forma extrapulmonar, principalmente ósea (5.8\%) y ganglionar $(5.8 \%)$. La mayor proporción $(78.9 \%)$ de los sujetos con la forma activa alcanzó $\geq 7$ puntos de los criterios de Stegen-Toledo, que fue diferente de los otros grupos $(p<0.01)$. Además, $40(76.9 \%)$ pacientes con tuberculosis activa tuvieron prueba positiva QFT-G ${ }^{\circledR}$ y sólo 33 (63.5\%) tuvieron dermorreacción positiva. Esto fue diferente $(p<0.01)$ en el grupo con tuberculosis latente pues sólo 9 (25.0\%) tuvieron QFT-G ${ }^{\circledR}$ positivo aunque 31 (86.11) fueron positivos al derivado proteínico purificado. Por otra parte, se observó que sólo $13(25.0 \%)$ de los pacientes con tuberculosis activa tuvieron la tríada de tos de más de dos semanas de evolución, fiebre y pérdida de peso. La radiografía de tórax mostró datos sugestivos de tuberculosis en 43 (82.7\%) de los pacientes con la forma activa y únicamente en dos (5.6\%) de los sujetos con la forma latente (Cuadro 3).
Estimación de la utilidad diagnóstica del ensayo de liberación

En general, el ensayo de liberación de interferón gamma tuvo sensibilidad de $76.2 \%$ (IC 95\%: $69.6,82.8$ ) y especificidad de $84.7 \%$ (IC $95 \%$ : $79.2,90.3)$ para identificar la infección con Mycobacterium tuberculosis; fue superior al derivado proteínico purificado que tuvo sensibilidad de $26.2 \%$ (IC 95\%: 17.4, 35.0) y especificidad de 56.6\% (IC 95\%: 48.6, 64.6). Para detectar tuberculosis activa la liberación de interferón tuvo sensibilidad de 76.9\% (IC 95\%: 69.5, 84.3), superior a la del derivado proteínico: $63.5 \%$ (IC 95\%: 53.8, 73.1) y esa diferencia (13.4\%) sí fue significativa. Esto no ocurrió con la especificidad, que fue muy semejante para ambas pruebas: 90.3 y $93.3 \%$, respectivamente. No se apreciaron diferencias en otros indicadores, excepto en la tasa de falsos negativos, que fue superior para la dermorreacción (36.5\%). No hubo diferencias significativas en los valores predictivos negativos.

En cuanto a la tuberculosis latente la sensibilidad del ensayo de liberación fue de sólo $25 \%$ (IC 95\%: 16.8, 33.2) mientras la del derivado proteínico fue $86.1 \%$ (IC 95\%: 79.2, 93.0); la diferencia entre éstos (61.1\%) fue estadísticamente significativa. No obstante, no hubo diferencia significativa en cuanto a especificidad con valores de 98.6 y $93.3 \%$, respectivamente.

Cuadro 2. Características biomédicas de niños con sospecha de infección tuberculosa de un hospital pediátrico del estado de Sonora (2012-2013)

\begin{tabular}{lcccc}
\hline \multirow{2}{*}{ Variable } & \multicolumn{3}{c}{ Media \pm error estándar } \\
& TBA $(\mathbf{n = 5 2})$ & TBL $(\mathbf{n = 3 6})$ & Controles $(\mathbf{n = 7 2})$ & $\boldsymbol{p}^{\mathbf{1}}$ \\
& $5.00 \pm 0.72$ & $0.26 \pm 0.11$ & $0.04 \pm 0.008$ & $<0.001$ \\
QFT-G $^{\circledR}(\mathrm{UI} / \mathrm{mL})^{\mathrm{A}}$ & $12.46 \pm 1.44$ & $14.86 \pm 1.65$ & $1.77 \pm 0.20$ & $<0.001$ \\
Dermorreacción al PPD (en milímetros) & $7.14 \pm 0.79$ & $5.81 \pm 0.77$ & $7.47 \pm 0.57$ & 0.275 \\
Edad (en años) & $27.628 \pm 2.599$ & $24.296 \pm 2.716$ & $29.633 \pm 2.494$ & 0.410 \\
Peso (en kg) & $1.19 \pm 0.05$ & $1.18 \pm 0.04$ & $1.22 \pm 0.03$ & 0.254 \\
Talla (en metros) & $17.31 \pm 0.40$ & $17.60 \pm 0.54$ & $16.57 \pm 0.51$ & 0.185
\end{tabular}

PPD: derivado proteínico purificado; QFT-G ${ }^{\circledR}$ : Quantiferon TB-Gold; TBA: tuberculosis activa; TBL: tuberculosis latente. ${ }^{1}$ Basado en una prueba de ANOVA de una sola vía.

${ }^{A}$ Expresado en unidades internacionales por mililitro. 
Cuadro 3. Características seleccionadas de niños con infección tuberculosa y un grupo control de un hospital pediátrico del estado de Sonora (2012 -2013)

\begin{tabular}{|c|c|c|c|c|}
\hline \multirow[t]{2}{*}{ Variable } & \multicolumn{3}{|c|}{ Número (\%) } & \multirow[t]{2}{*}{$p^{1}$} \\
\hline & TBA $(n=52)$ & TBL $(n=36)$ & Controles $(n=72)$ & \\
\hline \multicolumn{5}{|l|}{ Criterios Stegen-Toledo } \\
\hline$\geq 7$ puntos (diagnóstico de certeza de TB) & $41(78.85)$ & $1(2.78)$ & $0(00.00)$ & $<0.01$ \\
\hline 5-6 puntos (diagnóstico factible de TB) & $7(13.46)$ & $17(47.22)$ & $0(00.00)$ & 0.04 \\
\hline 3-4 puntos (diagnóstico posible de TB) & $4(7.69)$ & $11(30.56)$ & $7(9.72)$ & 0.186 \\
\hline 2 puntos (no es TB) & $0(00.00)$ & $7(19.44)$ & $65(90.28)$ & $<0.01$ \\
\hline \multicolumn{5}{|l|}{ QFT-G ${ }^{\circledR}$} \\
\hline Positivo ( $\geq 0.35 \mathrm{UI} / \mathrm{mL}$ ) & $40(76.92)$ & $9(25.00)$ & $1(1.39)$ & $<0.01$ \\
\hline Negativo & $12(23.08)$ & $27(75.00)$ & $71(98.61)$ & $<0.01$ \\
\hline \multicolumn{5}{|l|}{ PPD } \\
\hline Positivo ( $\geq 5$ mm diámetro) & $33(63.46)$ & $31(86.11)$ & $4(5.56)$ & $<0.01$ \\
\hline Negativo (< 5 mm diámetro) & $19(36.54)$ & $5(13.89)$ & $56(77.78)$ & $<0.01$ \\
\hline No se realizó & $0(0.00)$ & $0(0.00)$ & $12(16.67)$ & ns \\
\hline \multicolumn{5}{|l|}{ Cicatriz post-BCG } \\
\hline Sí & $50(96.1)$ & $35(97.2)$ & $71(98.6)$ & 0.002 \\
\hline No & $2(3.9)$ & $1(2.8)$ & $1(1.4)$ & 0.564 \\
\hline \multicolumn{5}{|l|}{ Contacto con TB ${ }^{\mathrm{A}}$} \\
\hline Sí & $40(76.92)$ & $22(61.11)$ & $1(1.39)$ & $<0.01$ \\
\hline No & $12(23.08)$ & $14(38.89)$ & $71(98.61)$ & $<0.01$ \\
\hline \multicolumn{5}{|l|}{ Triada clínica ${ }^{B}$} \\
\hline Presente & $13(25.00)$ & $0(0.00)$ & $1(1.39)$ & 0.001 \\
\hline Ausente & $39(75.00)$ & $36(100.0)$ & $71(98.61)$ & $<0.01$ \\
\hline \multicolumn{5}{|l|}{ Radiografía de tórax } \\
\hline Con datos sugestivos & $43(82.69)$ & $2(5.56)$ & $0(00.00)$ & $<0.01$ \\
\hline Sin datos sugestivos & $8(15.38)$ & $24(66.67)$ & $18(25.00)$ & 0.020 \\
\hline No se realizó & $1(1.92)$ & $10(27.78)$ & $54(75.00)$ & $<0.01$ \\
\hline
\end{tabular}

BCG: vacuna con bacilo de Calmette-Guerin; PPD: derivado proteínico purificado; QFT-G ${ }^{\circledR}$ : Quantiferon TB-Gold; TB: tuberculosis; TBA: tuberculosis activa; TBL: tuberculosis latente.

${ }^{1}$ Basada en una prueba multinomial de $\chi^{2}$.

${ }^{\text {AC }}$ Contacto sustancial ( $>4$ horas diarias) con casos de TB (intradomiciliar o extradomiciliar).

BPresentaron tos de dos o más semanas de evolución, fiebre y pérdida de peso.

Todos los indicadores evaluados se muestran en el Cuadro 4.

En lo referente a la confiabilidad con el ensayo de liberación de interferón gamma se obtuvo un coeficiente Kappa (K) de 0.57 (IC 95\%: 0.42, 0.73); cuando se estimó únicamente para pacientes con la forma activa de la enfermedad la K fue de 0.58 (IC 95\%: 0.42, 0.73) pero fue muy pobre y no significativa para pacientes con la forma latente ( $K=-0.03$, IC 95\%: -0.19 , 0.13). Para el derivado proteínico purificado el coeficiente fue muy bajo para cualquier forma de tuberculosis y en ninguna de ellas se apreció significación estadística.

\section{Relación del ensayo de liberación con predictores sociodemográficos y clínicos}

Tres variables clínicas predijeron significativamente una respuesta positiva del QFT-G ${ }^{\circledR}$ en el análisis factorial. La primera de ellas fue que el niño tuviera una radiografía posteroanterior de tórax con datos sugestivos de tuberculosis 
Cuadro 4. Indicadores de validez de dos métodos para el diagnóstico de tuberculosis en niños de un hospital pediátrico del estado de Sonora (2012-2013)

\begin{tabular}{|c|c|c|c|c|}
\hline Indicador & QFT-G $^{\circledR}$ & PPD & $\begin{array}{l}\text { Diferencia de } \\
\text { proporciones }\end{array}$ & IC $95 \%{ }^{1}$ \\
\hline \multicolumn{5}{|l|}{ Infección por Mt } \\
\hline Sensibilidad & $76.2(69.6,82.8)$ & $26.2(17.4,35.0)$ & 50.0 & $(38.4,61.6)^{* *}$ \\
\hline Especificidad & $84.7(79.2,90.3)$ & $56.6(48.6,64.6)$ & 28.1 & $(21.2,35.2)^{* *}$ \\
\hline Valor predictivo positivo & $64.0(56.6,71.4)$ & $19.3(11.9,26.7)$ & 44.7 & $(36.2,53.2)^{* *}$ \\
\hline Valor predictivo negativo & $90.9(86.5,95.4)$ & $65.9(57.0,74.9)$ & 25.0 & $(14.7,35.3)^{* *}$ \\
\hline Falsos positivos & $15.3(9.7,20.8)$ & $43.4(34.0,52.7)$ & 28.1 & $(21.1,35.1)^{* *}$ \\
\hline Falsos negativos & $23.8(17.2,30.4)$ & $73.8(65.5,82.1)$ & 50.0 & $(38.4,61.6)^{* *}$ \\
\hline Precisión & $82.5(76.6,88.4)$ & $48.0(38.6,57.4)$ & 34.5 & $(24.7,44.3)^{* *}$ \\
\hline \multicolumn{5}{|l|}{ Tuberculosis activa } \\
\hline Sensibilidad & $76.9(69.5,84.3)$ & $63.5(53.8,73.1)$ & 13.4 & $(2.0,25.0)^{* *}$ \\
\hline Especificidad & $90.3(85.1,95.5)$ & $93.3(88.7,98.0)$ & 3.0 & $(-4.0,10.0)$ \\
\hline Valor predictivo positivo & $85.2(78.8,91.4)$ & $89.2(83.3,95.0)$ & 4.1 & $(-4.0,13.0)$ \\
\hline Valor predictivo negativo & $84.4(78.0,90.8)$ & $74.7(66.5,82.9)$ & 9.7 & $(-1.0,20.0)$ \\
\hline Falsos positivos & $9.7(4.5,14.9)$ & $6.7(2.0,11.4)$ & 3.1 & $(-4.0,10.0)$ \\
\hline Falsos negativos & $23.1(15.7,30.5)$ & $36.5(27.5,45.6)$ & 13.5 & $(2.0,25.0)^{* *}$ \\
\hline Precisión & $84.7(78.3,91.0)$ & $79.5(71.8,87.1)$ & 5.2 & $(-5.0,15.0)$ \\
\hline \multicolumn{5}{|l|}{ Tuberculosis latente } \\
\hline Sensibilidad & $25.0(16.8,33.2)$ & $86.1(79.2,93.0)$ & 61.1 & $(0.50,0.72)^{* *}$ \\
\hline Especificidad & $98.6(96.4,100.0)$ & $93.3(88.3,98.3)$ & 5.3 & $(0.00,0.11)$ \\
\hline Valor predictivo positivo & $90.0(82.6,97.4)$ & $88.6(79.9,97.2)$ & 1.4 & $(-0.07,0.10)$ \\
\hline Valor predictivo negativo & $98.6(95.7,100.0)$ & $91.8(84.3,99.3)$ & 6.8 & $(-0.01,0.21)$ \\
\hline Falsos positivos & $1.4(0.0,4.3)$ & $6.7(0.0,13.4)$ & 5.3 & $(-0.04,0.11)$ \\
\hline Falsos negativos & $75(64.3,85.7)$ & $13.9(4.5,23.3)$ & 61.1 & $(0.49,0.74) * *$ \\
\hline Precisión & $74.1(63.3,84.9)$ & $90.6(82.7,98.5)$ & 16.6 & $(0.06,0.27)^{* *}$ \\
\hline
\end{tabular}

Mt: Mycobacterium tuberculosis; PPD: derivado proteínico purificado; QFT-G ${ }^{\circledR}$ : Quantiferon TB-Gold. ${ }^{1}$ Intervalo de confianza al $95 \%$ para la diferencia de dos proporciones muestrales.

**Diferencia estadísticamente significativa a 95\% de confianza.

(infiltrado alveolar tipo neumonía; derrame pleural, adenopatía hiliar o miliar) cuya razón de momios cruda (RMc) fue de 6.42 (IC 95\%: $2.62,15.72)$; la segunda fue la triada clínica con una RMc de 6.63 (IC 95\%: 1.97, 22.33) y la tercera fue la forma clínica de la tuberculosis: tanto la tuberculosis pulmonar (RMc 21.04, IC 95\%: 8.06, 54.93) como la extrapulmonar (RMc 21.04, IC 95\%: 4.70, 94.02) se asociaron con un QFT-G ${ }^{\circledR}$ positivo. De doce variables sociodemográficas y clínicas analizadas (edad, sexo, comorbilidades, desnutrición, cicatriz BCG, contacto con sospechoso de tuberculosis, municipio endémico, grupo étnico, ingreso familiar, ocupación y escolaridad de ambos padres, hacinamiento) sólo la última ( $\geq 2.5$ personas por habitación para dormir) se relacionó con un ensayo de liberación de interferón gamma positivo (RMc 2.53, IC 95\%: 1.13, 5.66).

En el análisis multivariado únicamente dos variables mantuvieron su valor para predecir un resultado positivo para el ensayo de liberación: los datos sugestivos de tuberculosis en la radiografía de tórax y la forma pulmonar de la tuberculosis. En el primer caso se observó que los sujetos con radiografía torácica sugestiva de tuberculosis tuvieron 4.4 veces (RM ajustada 4.37) más probabilidades de presentar ensayo de liberación positivo, lo que fue estadísticamente 
significativo. Para los sujetos con tuberculosis pulmonar se apreció que esa probabilidad fue 6.1 veces mayor (RM ajustada 6.15) en individuos con ensayo de liberación positivo, lo que también fue estadísticamente significativo. El modelo final mostró significación estadística $(p<0.01)$. Los resultados de la regresión logística multivariada se muestran en el Cuadro 5.

\section{DISCUSIÓN}

Nuestro estudio muestra que el ensayo de liberación de interferón gamma es un método válido para el diagnóstico de tuberculosis pediátrica y que puede emplearse como un recurso complementario para su detección en niños y adolescentes. El método identifica correctamente a 8 de cada 10 niños con Mycobacterium tuberculosis, aunque su utilidad es casi explicada totalmente por su capacidad para identificar la forma activa de la enfermedad. Esta capacidad es consistente con la reportada por otros estudios pediátricos, que oscila entre 66 y $83 \%$, aunque en países con economías en desarrollo esa sensibilidad fue sólo de $58 \% .^{10,11}$

La prueba parece tener mayor sensibilidad para detectar tuberculosis activa en países industrializados, fluctuando desde 50\% hasta 100\%; ${ }^{20,21}$ aunque otros reportes la ubican entre 70 y $85 \%$, algo menor al desempeño observado en adultos. ${ }^{10,11}$ En cambio, en naciones de medianos y bajos ingresos la sensibilidad del ensayo de liberación ha sido menor y oscila entre 58 y $80 \%,{ }^{10,11,22}$ un valor semejante al nuestro.

La capacidad del QFT-G ${ }^{\circledR}$ para identificar infección con M. tuberculosis no implica que pueda sustituir al derivado proteínico purificado como estrategia de cribado, por lo que una prueba positiva debe examinarse en los contextos clínico y epidemiológico específicos. Sin embargo, también es posible que un resultado negativo a la prueba pueda emplearse para descartar la enfermedad, especialmente en niños inmunocompetentes..$^{23}$ Por ello, es recomendable ejecutar estudios longitudinales para evaluar el valor predictivo negativo de la prueba, siempre que se considere la prevalencia del escenario en donde se efectúe la investigación. En tal sentido, la especificidad de la prueba QFT-G $^{\circledR}$ es un aspecto importante. En nuestro estudio la especificidad para tuberculosis activa fue $90.3 \%$, congruente con los resultados de estudios previos que documentaron especificidad entre 91 y 95\%. ${ }^{11,24}$ Así, el QFT-G ${ }^{\circledR}$ parece ser útil para detectar tuberculosis activa en regiones como la nuestra, especialmente en ausencia de

Cuadro 5. Regresión logística multivariada de predictores de la respuesta a QFT-G ${ }^{\circledR}$ en niños con sospecha de infección tuberculosa de un hospital pediátrico del estado de Sonora (2012-2013)

\begin{tabular}{lcccc}
\hline Variable & Coeficiente $\beta$ & Error estándar & RMa & IC 95\% \\
Intercepción & -2.142 & 0.438 & 0.12 & $(0.05,0.28)$ \\
Hacinamiento [1 = presente] & 0.318 & 0.500 & 1.37 & $(0.52,3.66)$ \\
Radiografía de tórax [1 = datos sugestivos de TB] & 1.474 & 0.573 & 4.37 & $(1.42,13.42)^{* *}$ \\
Triada clínica (tos, fiebre, pérdida de peso) [1 = Sí] & 0.638 & 0.786 & 1.89 & $0.41,8.83)$ \\
Forma de tuberculosis [Referencia = sin TB] & & & & \\
$\quad$ - TB pulmonar & 1.816 & 0.600 & 6.15 & $(1.90,19.92)^{* *}$ \\
$\quad$ - TB extrapulmonar & 1.167 & 0.873 & 3.21 & $(0.58,17.80)$ \\
$\quad$ - TB latente & 0.861 & 0.525 & 2.37 & $(0.85,6.62)$
\end{tabular}

**Estadísticamente significativo; QFT-G ${ }^{\circledR}$ : Quantiferon TB-Gold; TB: tuberculosis.

IC 95\%: intervalo de confianza a 95\%; RMa: razón de momios ajustada por las otras variables contenidas en el modelo. La significación general del modelo completo fue evaluada por $\chi^{2}=55.789, p<0.01$. 
confirmación bacteriológica como sucede con frecuencia en los casos pediátricos donde los datos clínicos y epidemiológicos, así como los hallazgos radiológicos, son los que apoyan el diagnóstico.

QFT-G ${ }^{\circledR}$ para el diagnóstico de la tuberculosis latente

En nuestro análisis el QFT-G ${ }^{\circledR}$ tuvo un comportamiento pobre para identificar niños con tuberculosis latente, algo ya descrito en otros trabajos. ${ }^{25}$ La sensibilidad que encontramos $(25 \%)$ es inferior a la publicada (55\%) por Machingaidze ${ }^{10}$ en niños de regiones con alta incidencia de tuberculosis. Kampmann y sus colaboradores ${ }^{26}$ señalaron una sensibilidad de $32 \%$ en niños vacunados o no con BCG. Este comportamiento del QFT-G ${ }^{\circledR}$ en niños es inferior al observado en adultos en quienes la sensibilidad fluctúa entre 61 y $90 \%$ para tuberculosis latente. ${ }^{11}$ Como resultado de esta sensibilidad limitada para la forma latente se ha señalado que no es mejor al derivado proteínico y que su utilidad se restringe a confirmar una historia de exposición a $M$. tuberculosis, que no distingue entre las formas activa y latente y puede ser obscurecida por infecciones remotas..$^{20}$

Contraria a su baja sensibilidad la prueba QFT$\mathrm{G}^{\circledR}$ mostró una excelente especificidad (98.6\%), lo que es consistente con otros reportes pediátricos. ${ }^{11,25,27}$ La especificidad del método para detectar tuberculosis latente sugiere que la prueba es útil para descartar la infección en niños; aunque los resultados deben ser interpretados cautelosamente ya que, ante la ausencia de un criterio de referencia apropiado para la forma latente y la escasez de estudios longitudinales, es difícil concluir acerca de su especificidad cuando se le compara con el derivado proteínico. ${ }^{20}$ La capacidad mostrada por el QFT-G ${ }^{\circledR}$ sugiere que su incorporación a los procedimientos diagnósticos empleados para descartar tuberculosis latente en niños tendría capacidad superior al derivado proteínico pues, entre otras cosas, no se ve afectado por las tasas de vacunación con BCG. ${ }^{27}$ Nuestro hallazgo puede ser útil pues la detección y tratamiento oportunos de la forma latente de la enfermedad tiene implicaciones médicas y sanitarias sustanciales: a) puede disminuir la progresión hacia la forma activa; b) un diagnóstico preciso y el tratamiento en niños puede prevenir la reactivación adulta; c) la tuberculosis activa en niños tiene relevancia epidemiológica pues evidencia transmisión activa de M. tuberculosis en la comunidad. ${ }^{10}$

\section{Concordancia del QFT-G ${ }^{\bullet}$ para detectar infección tuberculosa}

No tenemos conocimiento de estudios previos que usaran como estándar de referencia un sistema de puntuación que, si bien tiene limitaciones metodológicas y no existe consenso acerca de su validez, constituye una herramienta útil en regiones con limitaciones para la confirmación de la M. tuberculosis. Es posible, que en situaciones similares los criterios clínicos puedan emplearse razonablemente como estándar de referencia. Si esto es aceptable, entonces el QFT-G ${ }^{\circledR}$ muestra una confiabilidad moderada cuando se le compara con los criterios de Stegen-Toledo.

\section{Predictores de la positividad de QFT-G}

En nuestro estudio una radiografía de tórax con datos sugestivos de tuberculosis se asoció significativamente con la positividad del QFT-G ${ }^{\circledR}$. El porcentaje de concordancia entre ambos procedimientos fue moderado $(71.6 \%, \mathrm{~K}=0.432)$; algo que fue apoyado por el análisis multivariado pues un niño con rediografía sugestiva tuvo cuatro veces más probabilidad de tener una prueba positiva de QFT-G ${ }^{\circledR}$ una vez ajustado el efecto del hacinamiento, síntomas clínicos y la forma de enfermedad. Esta asociación fue mayor en la tuberculosis pulmonar: se incrementó seis veces la probabilidad de un QFT-G $^{\circledR}$ positivo. 
La relación que observamos entre QFT-G ${ }^{\circledR}$ y radiografía es distinta a la de otros informes ${ }^{27}$ en donde no se encontró ninguna asociación significativa. Nuestro hallazgo indicaría, por un lado, que la detección de la infección es tardía en los niños, lo que permitiría el desarrollo de la enfermedad hasta etapas avanzadas; así cobra relevancia el valor predictivo negativo (84.4\%) que muestra el QFT-G ${ }^{\circledR}$ para descartar tuberculosis activa. Por el otro, señala que la radiografía es un elemento importante del diagnóstico para detectar la forma activa de la enfermedad y que debe permanecer como una prueba de cribado de la tuberculosis en niños sintomáticos. La asociación observada debe ser tomada cautelosamente por una potencial colinearidad estadística entre la tuberculosis pulmonar y la radiografía torácica aunque, como señalamos, su hallazgo denota que la tuberculosis pulmonar no se sospecha oportunamente en la población pediátrica y que el QFT-G ${ }^{\circledR}$ puede ayudar a descartar su presencia dado su valor predictivo negativo.

Nuestro estudio tiene limitaciones: la primera es que la condición inmunitaria de los sujetos de estudio sólo fue investigada mediante el interrogatorio y la exploración médica, excepto para posible infección por VIH no se contó con controles positivos, lo que pudo dar falsos negativos en individuos con alguna inmunodepresión. Además, el antecedente de vacunación con BCG se comprobó únicamente con la identificación de la cicatriz característica, lo que pudo introducir errores de interpretación. La segunda limitación procede del criterio de referencia que empleamos pues existe un grado de subjetividad en la clasificación basada en los criterios clínicos, lo que podría sobrestimar la capacidad del QFT-G ${ }^{\circledR}$ para detectar infección tuberculosa. Además, es posible que el sesgo de selección del diseño elegido y la población que acude a atención hospitalaria tengan un efecto negativo en los resultados y que la capacidad diagnóstica del QFT-G $^{\circledR}$ esté sobrestimada. Es recomendable que se efectúen estudios cuya base poblacional incluya sujetos cuya condición clínica (de tuberculosis) aún no se encuentre en fases avanzadas.

\section{CONCLUSIONES}

Nuestros hallazgos indican que el ensayo de liberación de interferón gamma $\left(\mathrm{QFT}^{\circledR} \mathrm{G}^{\circledR}\right)$ es un método valioso para identificar la infección con Mycobacterium tuberculosis en niños y adolescentes atendidos en hospitales de regiones caracterizadas por exposición frecuente y con alta cobertura con BCG. La prueba parece particularmente útil para detectar la forma activa de la enfermedad (no tanto la latente) pues permite identificar a 8 de cada 10 niños con tuberculosis activa pero sólo a 3 de cada 10 con latente. Aparentemente posee alto valor predictivo negativo (> 90\%) y elevada especificidad (>90\%), lo que ayudaría a descartar correctamente a 9 de cada 10 niños sin infección con M. tuberculosis. El método posee suficiente validez clínica y puede ser útil porque apoyaría razonablemente la decisión de comenzar tratamiento antituberculoso en niños con sospecha de tuberculosis, lo que contribuiría favorablemente a reducir complicaciones médicas asociadas con un tratamiento tardío, particularmente de la forma pulmonar.

Finalmente, debe considerarse que un resultado positivo del QFT-G ${ }^{\circledR}$ puede reflejar infecciones recientes más que infecciones remotas o exposición frecuente al agente, lo que ayudaría al diagnóstico de la forma activa por la naturaleza antigénica de la prueba y porque la activación de la memoria de linfocitos T que produce sólo persiste por tiempo limitado. El QFT-G ${ }^{\circledR}$ puede ser una herramienta diagnóstica útil para tuberculosis en niños y adolescentes en quienes la enfermedad es notablemente difícil de confirmar bacteriológicamente. 


\section{Agradecimientos}

Esta investigación fue posible gracias al financiamiento del Fondo Sectorial CONACYT-SALUD (S0008-2010-1-139886). El equipo de trabajo expresa su particular gratitud a las Autoridades de la Secretaría de Salud Pública del Estado de Sonora por el apoyo brindado a la ejecución de este proyecto, así como al personal directivo, médicos residentes y nutriólogas del sitio de estudio, por su decidida participación en la fase de recolección de datos.

\section{REFERENCIAS}

1. World Health Organization. Diagnostics and laboratory strengthening. En: Global Tuberculosis Report 2014. Geneve, 2015. Disponible en línea en: http://apps.who.int/ iris/bitstream/10665/137094/1/9789241564809_eng.pdf

2. Secretaría de Salud. Centro Nacional de Programas Preventivos y Control de Enfermedades. Situación actual de tuberculosis en México y el mundo. XIX Curso de Actualización en el Diagnóstico y Tratamiento de la Tuberculosis en el Niño y en el Adulto. México, D.F. Junio 2015. Disponible en línea en: http://www.cenaprece.salud. gob.mx/programas/interior/micobacteriosis/descargas/ pdf/1SituacionEpidemiologicaTB.pdf

3. Hertting O, Shingadia D. Childhood TB: when to think of it and what to do when you do. J Infect 2014;68(Suppl 1):S151S154.

4. Nelson LJ, Dwells CD. Global epidemiology of childhood tuberculosis. Int J Tuberc Lung Dis 2004;8(5):636-647.

5. Swaminathan S, Rheka B. Pediatric tuberculosis: global overview and challenges. Clin Infect Dis 2010;50(S3):S184S194.

6. Nicol MP, Zar HJ. New specimens and laboratory diagnostics for childhood pulmonary TB: progress and prospects. Paediatr Resp Rev 2011;12(1):16-21.

7. Marais BJ, Pai M. Recent advances in the diagnosis of childhood tuberculosis. Arch Dis Child 2007;92(5):446-452 doi: 10.1136/adc.2006.104976

8. Shingadia D. The diagnosis of tuberculosis. Pediatr Infect Dis J 2012;31(3):302-305.

9. Pai M, Riley LW, Colford Jr. JM. Interferon- $\gamma$ assays in the immunodiagnosis of tuberculosis: a systematic review. Lancet Infect Dis 2004;4(12):761-776.

10. Machingaidze $S$, SheyWiysonge $C$, Gonzalez-Angulo $Y$, Hatherill M, Moyo S, Hanekom W, et al. The utility of an interferon gamma release assay for diagnosis of latent tuberculosis infection and disease in children. Pediatr Infect Dis J 2011;30(8):1-7.
11. Mandalakas AM, Detjen AK, Hesseling AC, Benedetti A, Menzies D. Interferon-gamma release assays and childhood tuberculosis: systematic review and meta-analysis. Int $J$ Tuberc Lung Dis 2011;15(8):1018-1032.

12. Garfein RS, Lozada R, Liu L, Laniado-Laborin R, Rodwell TC Deiss R, Alvelais J et al. High prevalence of latent tuberculosis infection among injection drug users in Tijuana, Mexico. Int J Tuberc Lung Dis 2009;13(5):626-632.

13. Burgos JL, Khan JG, Strathdee SA, Valencia-Mendoza A, Bautista-Arredondo S, Laniado-Laborin $\mathrm{R}$ et al. Targeted screening and treatment for latent tuberculosis infection using QuantiFERON ${ }^{\circledR}$-TB Gold is cost-effective in Mexico. Int J Tuberc Lung Dis 2009;13(8):962-968.

14. González-Salazar F, Vargas-Villarreal J, Garcialuna-Martínez FJ, Rivera G. Moreno-Treviño MG, Montfort-Gardeazabal JM et al. Snapshot of Quantiferon TB gold testing in Northern Mexico. Tuberculosis 2011;91(Suppl 1):S34-S37.

15. Howley MM, Painter JA, Katz DJ, Graviss EA, Reves R, Beavers SF et al. Evaluation of QuantiFERON-TB Gold InTube and tuberculin skin tests among immigrant children being screened for latent tuberculosis infection. Pediatr Infec Dis J 2015;34(1):35-39.

16. Castillo-Villela A. Valor diagnóstico de los criterios de Stegen modificado por Toledo en la Tuberculosis infantil en el Hospital Nacional Guillermo Almenara Irigoyen. Rev Peruan Pediatr 2005;58(2):30-39.

17. Mazurek GH, Jereb J, LoBlue P, lademarco MF, Metchcock B, Vernon A. Guidelines for using the QuantiFERON ${ }^{\circledR}$ TB-Gold test for detecting Mycobacterium tuberculosis infection, United States. MMWR 2005;54(RR15):49-55.

18. Pediatric Tuberculosis Collaborative Group. Targeted tuberculin skin testing and treatment of latent tuberculosis infection in children and adolescents. Pediatr 2004;114(Suppl 4):1175-1201.

19. Landis RJ, Koch GC. The measurement of observer agreement for categorical data. Biometrics 1977;33(1):159-174.

20. Kakkar F, Allen UD, Ling D, Pai M, Kitai IC. Tuberculosis in children: new diagnostic blood tests. Paediatr Child Health 2010;15(8):529-533.

21. Bianchi L,Galli L, Moriondo M, Veneruso G, Becciolini L,Azzari Ch et al. Interferon-Gamma release assay improves the diagnosis of tuberculosis in children. Pediatr InfectDis J 2009;28(6):510-514.

22. Blandinieres A, de Lauzanne A, Guerin-El Khourouj V, Gourgouillon N, See H, Pedron B, et al. QuantifERON to diagnose infection by Mycobacterium tuberculosis: Performance in infants and older children. J Infect 2013;67(5):391-398.

23. Pai $\mathrm{M}$, Joshi $\mathrm{R}$, Kalantri $\mathrm{S}$. Diagnosis of latent tuberculosis infection: recent advances and future directions. In: Tuberculosis. Sharma SK \& Mohan A (Eds). Jaypee Bros. Medical Publishers. New Delhi, 2009. pp. 186-199.

24. Chiappini E, Accetta G, Bonsignori F, Boddi V, Galli L, Biggeri $\mathrm{A}$, et al. Interferon- $\gamma$ release assays for the diagnosis of $\mathrm{My}$ cobacterium tuberculosis infection in children: a systematic 
review and meta-analysis. Int J Immunopathol Pharmacol 2012;25(3):557-564.

25. Okada K, Mao TE, Mori T, Miura T, Sugiyama T, Yoshima T et al. Performance of an interferon-gamma release assay for diagnosing latent tuberculosis infection in children. Epidemiol Infect 2008;136(9):1179-1187.

26. Kapmann B, Whittaker E, Williams A, Walters S, Gordon $A$, Martinez-Alier $N$ et al. Interferon- $\gamma$ release assays do not identify more children with active tuberculosis than the tuberculin skin test. Eur Respir J 2009;33(6):13741382.

27. Diel R, Goletti D, Ferrara G, Bothamley G, Cirillo D, Kampmann B et al. Interferon- $\gamma$ release assays for the diagnosis of latent Mycobacterium tuberculosis infection: a systematic review and meta-analysis. Eur Respir J 2011;37(1):88-99. DOI: 10.1183/09031936.00115110.

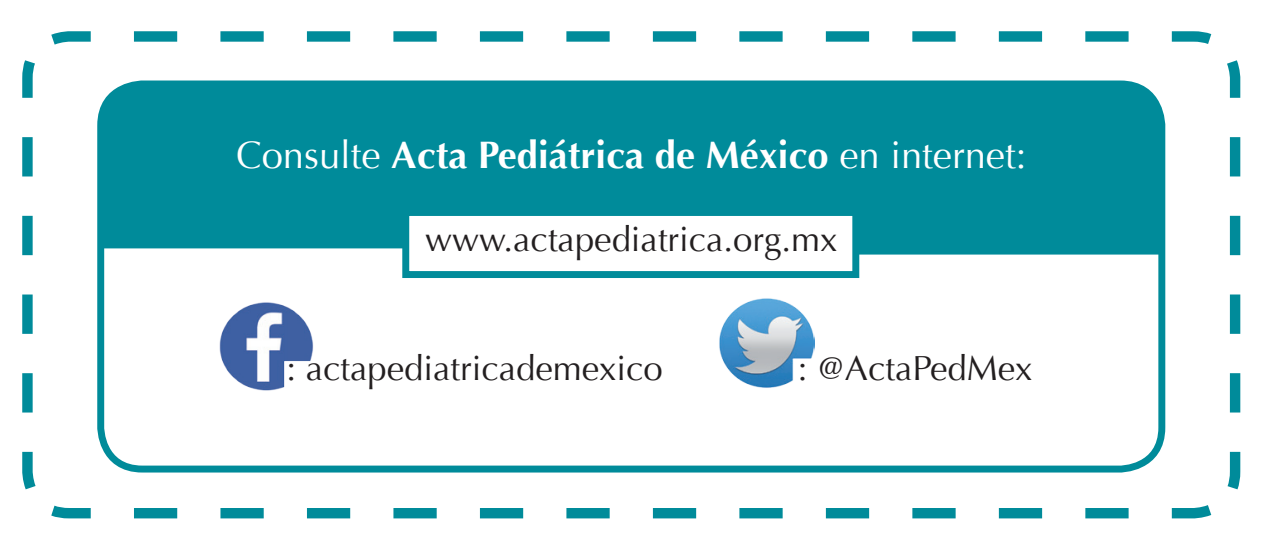

